\title{
РУССКОЯЗЫЧНАЯ ВЕРСИЯ ШКАЛЫ ОЦЕНКИ ОТНОШЕНИЙ
}

\author{
O.А. СЫЧЕВ \\ a ФГБОУ ВПО «Алтайская государственная академия образования имени В.М. Нукшина», \\ 659333, Россия, Алтайский край, Бийск, ул. Короленко, д. 53
}

\begin{abstract}
Резюме
В статье представлены результаты разработки русскоязычной версии Шкалы оценки отношений С. Хендрик. В отличие от известных в нашей стране тестов удовлетворенности браком данная методика характеризуется удобством в применении, экономичностью и пригодностью для диагностики удовлетворенности как супружескими, так и другими близкими отношениями взрослых. Апробация предложенной русскоязычной версии проводилась на трех выборках (общим объемом $\mathrm{N}=602$ ), включающих студентов, сотрудников бюджетных организаций и пользователей Интернета разного возраста (проходивших исследование в форме онлайн-тестирования), состоящих в официальном браке или в добрачных отношениях. Результаты конфирматорного факторного анализа подтвердили одномерную структуру методики (с наличием ковариации, отражающей особенности реагирования испытуемых на обратные пункты шкалы). Методика показала отличную надежность по внутренней согласованности (альфа Кронбаха $0.84-0.89$ ) и ретестовую надежность (более $0.70)$. Конвергентная валидность подтверждается высокими корреляциями с двумя методиками диагностики удовлетворенности браком ( $\mathrm{r}=0.82$ и 0.88$)$. В пользу конструктной валидности свидетельствует наличие предсказуемых корреляций со шкалами стилей любви, привязанности, субъективного благополучия и счастья. Выявлена умеренная связь результатов шкалы со склонностью к самообману. Значимое влияние на удовлетворенность отношениями показали факторы пола, семейного положения и их взаимодействие, причем наибольшей уровень удовлетворенности оказался характерен для мужчин, состоящих в браке. Хотя удовлетворенность отношениями у мужчин значимо выше, чем у женщин, величина различий невелика (d Коэна $=0.25)$. Анализ дифференциального функционирования пунктов шкалы показал их независимость от пола при наличии связи с семейным положением, что может отражать разную интерпретацию некоторых пунктов лицами, состоящими в отношениях разного типа. Полученные результаты позволяют рекомендовать предложенную версию методики в качестве инструмента оценки удовлетворенности супружескими или иными близкими отношениями взрослых.
\end{abstract}

Ключевые слова: удовлетворенность отношениями, шкала оценки отношений, стили любви, стили привязанности, субъективное благополучие.

Исследование выполнено при поддержке РГНФ, проект № 15-16-22003 «Социально-демографические и психологические факторы удовлетворенности отношениями в молодых семьях Алтайского края». 


\section{Теоретические основы исследования}

В психологических исследованиях супружеских отношений, как правило, одно из центральных мест занимает проблема удовлетворенности супругов браком. Выступая в качестве одного из важнейших индикаторов благополучия семьи, удовлетворенность супругов связана с устойчивостью брака, что свидетельствует о важности этой проблемы для решения практических задач в области психологического консультирования и социальной поддержки семьи. Хотя супружеские отношения во многом отличаются от других романтических отношений (например, добрачных), их психологические истоки, включающие, в первую очередь, чувства любви и привязанности к партнеру, являются общими. Следовательно, разумной представляется попытка общего подхода к определению и оценке удовлетворенности романтическими отношениями, основанного на представлениях об их единой психологической сущности независимо от стадии развития отношений (добрачной, супружеской) или их формы (официальный брак, незарегистрированный брак, гостевой брак и пр.).

Подобный подход предложили С. Хендрик и К. Хендрик, которые определяют удовлетворенность отношениями через субъективные переживания партнеров по поводу их взаимоотношений, в отличие от адаптации (adjustment), проявляющейся на уровне поведения и взаимодействия друг с другом (Hendrick, Hendrick, 1997). По мнению этих авторов, супруги могут демонстриро- вать достаточно хорошую взаимную адаптацию, проявляющуюся в согласии по поводу важных семейных функций и отсутствии конфликтов, но при недостаточном удовлетворении их эмоциональных потребностей удовлетворенность отношениями может быть невысока. В то же время встречаются относительно удовлетворенные своими отношениями пары, имеющие глубокую эмоциональную близость, которые при этом могут не находить согласия по довольно важным вопросам и спорить, демонстрируя не слишком успешное приспособление друг к другу на поведенческом уровне. В основу Шкалы оценки отношений было положено такое понимание удовлетворенности отношениями, которое акцентирует внимание на субъективном эмоциональном опыте взаимоотношений, отражающем удовлетворенность психологических потребностей партнеров.

Удовлетворенность отношениями представляет интерес для психологов, прежде всего, ввиду того, что она оказывает существенное влияние на развитие отношений в будущем (Hendrick, 1988). Вместе с тем важно учитывать тот факт, что удовлетворенность не является единственным фактором, определяющим развитие отношений: даже в парах с относительно высокой удовлетворенностью отношения могут прекратиться при недостаточной мотивации к их сохранению. Напротив, при некоторых обстоятельствах весьма стабильными оказываются пары с относительно низкой удовлетворенностью (Rusbult, Buunk, 1993). Удовлетворенность отношениями демонстрирует связь с общей удовлетворенностью 
жизнью (Scorsolini-Comin, dos Santos, 2012), а также с показателями соматического и психического здоровья (Guerrero et al., 2011). Наконец, удовлетворенность отношениями может представлять интерес как самостоятельный показатель психологического благополучия и удовлетворенности жизнью в одной из ее важнейших сфер - близких отношениях.

К числу наиболее важных факторов удовлетворенности отношениями относятся, в первую очередь, особенности любви и привязанности. Еще тридцать лет назад в статье, посвященной описанию самой известной отечественной методики диагностики удовлетворенности браком, ее авторы констатировали: «Достаточно доказанным является тезис о том, что основным фактором, скрепляющим супружеские союзы, являются эмоциональные узы любовь, эмоциональная привязанность» (Столин и др., 1984, с. 54). Многочисленные факты в подтверждение этого тезиса были получены в исследованиях, проведенных зарубежными психологами в последние десятилетия.

Значительный вклад в исследование любви как фактора удовлетворенности отношениями принадлежит С. Хендрик и К. Хендрик, развивающим концепцию стилей любви Дж.А. Ли. В проведенном ими обзоре приводится множество данных о том, что для мужчин и женщин различных национальностей, состоящих в браке с различным стажем отношений или состоящих в добрачных отношениях, стили любви являются весьма существенным фактором удовлетворенности отношениями
(Hendrick, Hendrick, 1997). О значении стилей любви говорит тот факт, что их вклад в удовлетворенность отношениями, как правило, превышает вклад различных свойств личности. Другой существенный фактор удовлетворенности отношениями стили романтической привязанности взрослых. Обобщение результатов исследований свидетельствует о том, что наиболее высокие оценки отношений демонстрируют лица с надежным стилем привязанности, в то время как лицам с амбивалентным и избегающим стилями в целом свойственна значительно меньшая удовлетворенность (Koski, Shaver, 1997).

В нашей стране при исследовании благополучия супружеских отношений обычно использовалось понятие удовлетворенности браком, причем в качестве его основных факторов рассматривались личностные особенности и психологическая совместимость супругов, согласованность их ценностей, ролевых ожиданий, стереотипов и установок (Андреева, 2005; Сопун, Лидерс, 2007). В подобных исследованиях чаще всего применяются такие методики, как «Опросник удовлетворенности браком», разработанный В.В. Столиным, Т.А. Романовой, Г.П. Бутенко (Столин и др., 1984), и «Тест на удовлетворенность браком» Ю.Е. Алешиной, Л.Я. Гозмана и Е.М. Дубовской (Алешина и др., 1987). Разработанные в середине 1980-х гг., эти опросники до сих пор составляют основной инструментарий в исследованиях супружеских отношений. К числу их несомненных достоинств можно отнести довольно высокую надежность и валидность, относительную 
простоту проведения и обработки результатов, доступность информации и наличие большого числа опубликованных работ. Вместе с тем можно указать и на некоторые общие недостатки этих методик. Сравнительно большое число заданий для одномерной шкалы (24 в опроснике В.В. Столина и 16 в тесте Ю.Е. Алешиной) в сочетании с не слишком удобной формой заданий требует лишних затрат времени и усилий со стороны испытуемого, затрудняя использование методик в составе объемных тестовых батарей. Формулировки заданий таковы, что эти методики оказываются малопригодны для исследования отношений, выходящих за рамки официального брака. Несмотря на длительный опыт использования опросника Столина в нашей стране, до настоящего времени недостаточно данных относительно статистических норм по этой методике. Таким образом, актуальной задачей является разработка новой методики, не уступающей рассмотренным по психометрическим характеристикам и лишенной их недостатков.

\section{Характеристика методики}

Шкала оценки отношений была предложена С. Хендрик в 1988 г. для оценки удовлетворенности отношениями супружеских пар и пар, не состоящих браке (Hendrick, 1988). Данная методика включает 7 заданий, каждое из которых предполагает оценку по пятибалльной шкале Лайкерта (см. в приложении: Шкала оценки отношений). В некоторых публикациях упоминаются версии этой методики с семибалльной шка- лой ответов, однако такой вариант является значительно менее распространенным (Dinkel, Balck, 2005).

Шкала оценки отношений была адаптирована в разных странах, так что в настоящее время можно обнаружить сведения о версиях этой методики на немецком, португальском и шведском языках (Dinkel, Balck, 2005; Cassepp-Borges, Pasquali, 2011; Rask et al., 2010). Результаты разных версий шкалы оценки отношений показывают умеренные и высокие корреляции с тестами, оценивающими удовлетворенность супругов браком, приемлемую ретестовую надежность и устойчивые психометрические показатели в группах испытуемых разной национальности и возраста. Для всех версий методики были получены высокие оценки надежности по внутренней согласованности (в пределах .85-.89). Для оригинальной версии шкалы, по данным С. Хендрик (1988), коэффициент надежности по внутренней согласованности ( $\alpha$ Кронбаха) составляет .86, а корреляции заданий со шкалой лежат в пределах от .57 до .76. Наиболее убедительным свидетельством конвергентной валидности данной методики является высокая корреляция с показателями по шкале адаптации в диадических отношениях (DAS) (Hendrick, 1988; Vaughn, Matyastik Baier, 1999).

Хорошая прогностическая валидность шкалы была показана в исследовании С. Хендрик (1988) на 30 добрачных парах. Результаты исследования свидетельствуют о высокой точности прогноза развития отношений спустя три месяца после тестирования (для пар, сохранивших отношения, точность прогноза 
составляла 91\%, для расставшихся пар - 86\%). Это означает, что шкала удовлетворенности отношениями не только характеризует текущую удовлетворенность, но и в некоторой мере позволяет предсказать стабильность отношений в будущем. Косвенно о хороших психометрических характеристиках методики также свидетельствует тот факт, что в парах обычно обнаруживается высокая корреляция между удовлетворенностью отношениями у партнеров (Hendrick, Hendrick, 1997).

Данная методика разрабатывалась автором как одношкальная, и в большинстве исследований, выполненных с помощью факторного анализа, подтверждается именно такая ее структура. Иногда можно обнаружить сведения о возможных двухфакторных решениях. Например, на шведской версии шкалы удовлетворенности отношениями было выявлено два фактора, которые были интерпретированы как «Отношения, основанные на удовлетворении потребностей» и «Отношения, основанные на любви и преданности» (Rask et al., 2010). Тем не менее бо́льшая часть эмпирических исследований на различных версиях методики подкрепляет вывод об однофакторной структуре методики.

Таким образом, к числу достоинств этой методики можно отнести, во-первых, ее направленность на оценку удовлетворенности отношениями партнеров, которые могут состоять или не состоять в браке. Гибкость методики делает возможным ее использование для оценки различных отношений, не укладывающихся в представления о браке в юридическом смысле (например, добрачные отношения, незарегистрированный брак, отношения в ЛГБТ-парах и пр.). Более того, после небольшой модификации утверждений (Renshaw et al., 2011) была предложена новая версия методики (RAS-G), пригодная для оценки удовлетворенности не только романтическими, но и любыми другими отношениями с окружающими людьми. Во-вторых, краткость и экономичность в отношении затрат времени на проведение и обработку результатов также являются важными плюсами методики. В-третьих, отличные психометрические показатели данной шкалы гарантируют высокую достоверность результатов. В-четвертых, распространенность методики в разных странах и наличие адаптаций на разных языках открывают новые возможности кросс-культурных исследований. Рассмотренные достоинства шкалы оценки отношений убеждают в актуальности работы по адаптации данной методики.

\section{Организация и методы исследования}

Целью исследования стала разработка русскоязычной версии Шкалы оценки отношений. Разрешение на адаптацию Шкалы оценки отношений и ее использование в исследованиях было получено от проф. С. Хендрик. На первом этапе оригинальный текст методики был переведен на русский язык автором статьи без изменений. Оценка точности перевода проводилась с использованием обратного перевода, выполненного экспертом, одинаково хорошо владеющим русским и английским языком. Для полученной в итоге шкалы 
был проведен анализ надежности и факторной структуры, направленный на подтверждение ее единства и внутренней согласованности.

Задачей следующего этапа исследования стала проверка конвергентной и конструктной валидности шкалы. Чтобы показать конвергентную валидность, результаты Шкалы оценки отношений было необходимо сопоставить с результатами других методик, измеряющих тот же самый конструкт. Поскольку в супружеских парах удовлетворенность отношениями, по существу, совпадает с удовлетворенностью браком, для решения этой задачи были выбраны две методики: Опросник удовлетворенности браком (ОУБ), разработанный В.В. Столиным, Т.А. Романовой, Г.П. Бутенко (1984), и Тест на удовлетворенность браком Ю.Е. Алешиной, Л.Я. Гозмана и Е.М. Дубовской (1987).

Анализ конструктной валидности был направлен на проверку гипотез о связи удовлетворенности отношениями с другими психическими явлениями. С учетом рассмотренных выше результатов исследований основных факторов удовлетворенности отношениями было выдвинуто предположение о тесной связи удовлетворенности с особенностями любви и привязанности. Для их диагностики были выбраны соответствующие методики, представляющие собой русскоязычные версии известных зарубежных тестов, что позволило не только проверить гипотезу, но и сопоставить полученные результаты с зарубежными данными. Для оценки выраженности чувств любви и симпатии к партнеру использовалась методика Шкалы любви и симпатии 3. Рубина в адап- тации Ю.Е. Алешиной, Л.Я. Гозмана (1987). Диагностика стилей любви проводилась с помощью методики Шкала стилей любви (Love Attitude Scale) К. Хендрик и С. Хендрик в адаптации О.А. Екимчик (2009). Диагностика стилей привязанности была выполнена с помощью методики Опыт близких отношений (Revised Experiences in Close Relationships Measure of Adult Attachment) К. Бреннан и Р.К. Фрейли, адаптированной Т.В. Казанцевой (2008).

С учетом данных о вкладе удовлетворенности отношениями в общую удовлетворенность жизнью (Scorsolini-Comin, dos Santos, 2012) в ходе анализа валидности была проверена гипотеза о связи между этими переменными. Для оценки удовлетворенности жизнью использовались Шкала счастья С. Любомирски в адаптации Е.Н. Осина, Д.А. Леонтьева (2008) и Шкала субъективного благополучия А. Бадо, Г.А. Мендельсон в адаптации М.В. Соколовой (1996).

После исследования валидности методики на следующем этапе был проведен анализ подверженности результатов шкалы влиянию установки на социально желательные ответы. Для решения этой задачи использовался Опросник социальной желательности BIDR (Balanced Inventory of Desirable Responding, адаптация Е.Н. Осина, 2011).

Для получения норм к тесту и оценки возможностей использования методики в разных совокупностях был проведен анализ различий в удовлетворенности отношениями в зависимости от демографических характеристик. Чтобы уточнить возможность сопоставления результатов в разных группах, также был выпол- 
нен DIF-анализ (Differential item functioning, анализ дифференциального функционирования пункта) заданий шкалы в зависимости от пола и семейного положения (Фер, Бакарак, 2010; Wang, Wang, 2012).

В исследовании психометрических характеристик русскоязычной версии методики приняли участие 602 испытуемых. Большинство испытуемых (студенты АГАО им. B.M. Шукшина и работники бюджетных организаций г. Бийска) проходили тестирование в бланковой форме. Кроме того, проводилось интернет-тестирование, к участию в котором через тематические форумы и группы в социальных сетях приглашались только лица, состоящие в браке. Подробная количественная характеристика выборок приведена в таблице 1.

В каждой из трех выборок Шкала оценки отношений показала высокую надежность по внутренней согласованности (в выборке студентов - .84; работников бюджетных организаций - .85; интернет-выборке -.89$)$. Этот факт свидетельствует о том, что надежность практически не зависит от способа предъявления и шкала функционирует одинаково в разных группах. Средние значения по шкале оценки отношений в разных выборках лежат в пределах от 3.69 (в интернет-выборке) до 3.90 (в выборке студентов) и не показывают статистически значимых различий.

Хотя данные выборки существенно различаются по некоторым важным характеристикам (возрастному и половому составу, типу отношений), влияние таких особенностей на удовлетворенность отношениями, как показано далее, является довольно слабым или вовсе отсутствует. В отношении других характеристик (таких как уровень образования, религиозность) эти группы не показывают принципиальных отличий. Неудивительно, что между выборками не обнаруживается статистически значимых различий в удовлетворенности отношениями. Все это позволяет надеяться, что, несмотря на довольно разный состав выборок, их объединение не угрожает достоверности результатов.

\section{Результаты}

\section{Надежность и факторная структура методики}

Коэффициент $\alpha$ Кронбаха составил .86 ( $\mathrm{N}=602)$, средняя межпози-

Характеристика выборок испытуемых

Таблица 1

\begin{tabular}{|l|c|c|c|c|}
\hline \multicolumn{1}{|c|}{ Выборка } & $\mathbf{N}$ & $\begin{array}{c}\text { Количество } \\
\text { мужчин (\%) }\end{array}$ & $\begin{array}{c}\text { Количество лиц, со- } \\
\text { стоящих в браке (\%) }\end{array}$ & $\begin{array}{c}\text { Средний } \\
\text { возраст } \pm \boldsymbol{\sigma}\end{array}$ \\
\hline Студенты АГАО & 108 & $29(27 \%)$ & $0(0 \%)$ & $19.44 \pm 1.94$ \\
\hline $\begin{array}{l}\text { Работники бюджетных } \\
\text { организаций }\end{array}$ & 313 & $105(34 \%)$ & $177(57 \%)$ & $28.01 \pm 8.47$ \\
\hline Интернет-выборка & 181 & $20(11 \%)$ & $181(100 \%)$ & $28.54 \pm 8.78$ \\
\hline Вся выборка & 602 & $176(29 \%)$ & $358(60 \%)$ & $26.63 \pm 8.51$ \\
\hline
\end{tabular}


ционная корреляция равна .47, что очень близко к значениям, полученным на зарубежных версиях методики. Корреляции заданий со шкалой лежат в пределах от .47 до .73. Эти показатели свидетельствуют об отличной внутренней согласованности русскоязычной версии шкалы.

Проверка ретестовой надежности проводилась на выборке в 48 испытуемых (студентов факультета психологии АГАО, состоящих в добрачных отношениях) с интервалом в три недели. Коэффициент корреляции составил .78 $(p<.001)$, что свидетельствует о достаточно высокой устойчивости результатов в течение этого срока. В другой выборке из 42 студенток, состоящих в добрачных отношениях, была показана достаточно высокая ретестовая надежность в течение длительного временно́го периода (четырех месяцев). Значение коэффициента надежности составило .74 $(p<.001)$.

Для анализа факторной структуры методики сначала был проведен эксплораторный факторный анализ с помощью метода главных компонент. На основе анализа графика собственных значений (по критерию осыпи) наиболее предпочтительным оказалось однофакторной решение. Полученный в результате общий фактор объясняет 55\% дисперсии заданий методики, при этом факторные нагрузки каждого задания имеют значения не ниже .59.

Далее соответствие структуры методики однофакторной модели было проверено с помощью конфирматорного факторного анализа. Исходной стала модель с одним фактором удовлетворенности отношениями без учета ковариаций ошибок.
Конфирматорный факторный анализ выполнялся в пакете Mplus 6 с использованием алгоритма оценки MLR, устойчивого к отклонениям от нормального распределения.

По результатам анализа индексов модификации в модель была внесена ковариация между ошибками двух обратных заданий (пункты № 4 и 7). Необходимо отметить, что вмешательство фактора формы заданий (прямой или обратной) является типичным для подобных шкальных методик и, как правило, учитывается в модели либо посредством введения ковариаций (как в нашем случае), либо включением в модель дополнительного фактора, соответствующего обратной форме заданий. Полученная в результате модель (см. рисунок 1) продемонстрировала удовлетворительные показатели соответствия исходным данным: $\chi^{2}=53.70$; $\mathrm{df}=13$; $p<.001$; RMSEA $=.072 ; 90 \%$-й доверительный интервал для RMSEA = $.053-.093 ; \mathrm{CFI}=.965 ;$ TLI $=.943$. Эти значения позволяют сделать вывод о подтверждении однофакторной структуры Шкалы оценки отношений.

\section{Конвергентная валидность методики}

Результаты оценки конвергентной валидности с использованием в качестве внешних критериев двух наиболее известных отечественных методик диагностики удовлетворенности браком на выборке испытуемых, состоящих в официальном браке, представлены в таблице 2.

Коэффициенты корреляции между всеми тремя методиками, измеряющими удовлетворенность отно- 


\section{Факторная структура Шкалы оценки отношений}

Рисунок 1

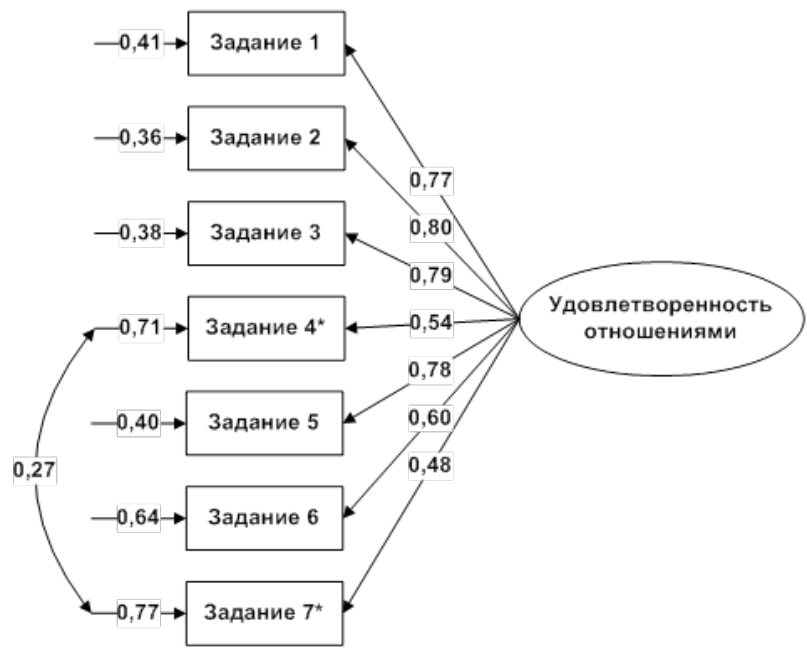

* - оценки по обратным заданиям 4 и 7 инвертированы.

Таблииа 2

Коэффициенты корреляции Пирсона показателей удовлетворенности браком

и Шкалы оценки отношений $(\mathrm{N}=168)$

\begin{tabular}{|c|c|c|c|}
\hline Методика & ШОО & ОУБ & ТУБ \\
\hline ШОО & $(.86)$ & & \\
\hline ОУБ & $.82^{* * *}$ & $(.88)$ & $(.91)$ \\
\hline ТУБ & $.88^{* * *}$ & $.87^{* * *}$ & \\
\hline
\end{tabular}

$* * * p<.001$.

Примечание. В скобках приведены значения коэффициентов надежности $\alpha$ Кронбаха; ШОО - Шкала оценки отношений С. Хендрик; ОУБ - Опросник удовлетворенности браком В.В. Столина, Т.А. Романовой, Г.П. Бутенко; ТУБ - Тест на удовлетворенность браком Ю.Е. Алешиной, Л.Я. Гозмана и Е.М. Дубовской.

шениями и браком, оказались весьма высоки (от .82 до .88 при уровне значимости $p<.001)$, свидетельствуя об их содержательной близости и взаимозаменяемости. Этот факт позволяет с большой уверенностью говорить о конвергентной валидности Шкалы оценки отношений.

Приведенные в таблице данные также свидетельствуют о том, что надежность всех трех методик находится приблизительно на одном уровне, хотя Шкала оценки отношений включает в себя значительно меньшее число заданий (величина коэффициента внутренней согласованности альфа Кронбаха при прочих равных условиях увеличивается по мере роста количества заданий в шкале). Таким образом, можно сде- 
лать вывод о том, что Шкала оценки отношений выступает как экономичное средство диагностики удовлетворенности отношениями, не уступающее по своим психометрическим характеристикам общепринятым в нашей стране методикам диагностики удовлетворенности браком.

\section{Связь любви и привязанности с удовлетворенностью отношениями}

Учитывая тесную связь удовлетворенности отношениями с чувством любви, анализ конструктной валидности методики разумно начать именно с исследования связей показателей по шкале оценки отношений со шкалами любви. Хотя в зарубежной психодиагностике известно очень много методик, предназначенных для измерения различных аспектов и проявлений любви (упоминаются 33 подобные методики - Hatfield et al., 2012), российские психологи располагают значительно меньшим инструментарием. Из числа зарубежных тестов любви успешно адаптированы и приобрели широкую известность у нас в стране шкалы любви и привязанности 3. Рубина (в адаптации Ю.Е. Алешиной и Л.Я Гозмана). Кроме того, неоднократно предпринимались попытки разработать методику диагностики стилей любви, основанную на типологии стилей любви Дж.А. Ли, получившей широкую известность в нашей стране благодаря работам Ю.Б. Рюрикова (1997). В решении этой задачи, повидимому, наиболее успешный результат получен О.А. Екимчик, предложившей русскоязычную версию шкалы стилей любви С. Хендрик и К. Хендрик (Екимчик, 2009).
Одним из недочетов этой версии стало то, что все шкалы, за исключением одной, показывают относительно невысокую надежность по внутренней согласованности (менее .7) в сравнении как с общепринятыми в психодиагностике требованиями, так и с оригинальной версией методики. В этой связи в данном исследовании использовалась несколько модифицированная версия методики, в которой отдельные утверждения были переформулированы таким образом, чтобы приблизить их содержание к оригинальной англоязычной версии и тем самым повысить внутреннюю согласованность шкал. Приведенные в таблице значения коэффициентов альфа Кронбаха свидетельствуют о том, что для большинства шкал эта цель была достигнута. Следовательно, несмотря на то, что существует необходимость в дополнительных исследованиях по уточнению факторной структуры и валидности русскоязычной версии методики, ее можно с известной осторожностью использовать в исследовательских целях.

Анализ корреляций, приведенных в таблице 3, показывает, что любовь действительно является весьма существенным фактором удовлетворенности отношениями. В частности, тесные связи с оценкой отношениями показала «Шкала любви», стиль любви «Эрос» и «Шкала симпатии». Кроме того, умеренная связь на высоком уровне значимости была выявлена со стилем любви «Агапе». Именно стили «Эрос» и «Агапе» в наибольшей мере соответствуют стереотипу подлинной романтической любви, так что связь этих стилей, так же как шкал любви и симпатии, с 
Таблица 3

Коэффициенты корреляции шкалы оценки отношений со шкалами любви и симпатии 3. Рубина и стилями любви

\begin{tabular}{|l|l|c|c|}
\hline \multicolumn{1}{|c|}{ Методика } & \multicolumn{1}{|c|}{ Шкала } & $\begin{array}{c}\text { Шкала оценки } \\
\text { отношений }\end{array}$ & $\begin{array}{c}\text { Коэффициенты надежности } \\
\text { шкал }(\boldsymbol{\alpha} \text { Кронбаха) }\end{array}$ \\
\hline \multirow{4}{*}{$\begin{array}{l}\text { Шкалы Рубина } \\
(\mathrm{N}=171)\end{array}$} & Шкала любви & $.61^{* * *}$ & .74 \\
\cline { 2 - 4 } & Шкала симпатии & $.49^{* * *}$ & .64 \\
\hline \multirow{4}{*}{$\begin{array}{l}\text { Шкалы стилей } \\
(\mathrm{N}=285)\end{array}$} & Эрос & $.53^{* * *}$ & .75 \\
\cline { 2 - 4 } & Агапе & $.32^{* * *}$ & .80 \\
\cline { 2 - 4 } & Сторге & $.24^{* * *}$ & .77 \\
\cline { 2 - 4 } & Мания & -.03 & .77 \\
\cline { 2 - 4 } & Прагма & $-.12^{*}$ & .69 \\
\cline { 2 - 4 } & Людус & $-.25^{* * *}$ & .62 \\
\hline
\end{tabular}

${ }^{*} p<0.05, * * * p<0.001$.

удовлетворенностью отношениями полностью соответствует тезису о том, что удовлетворенность отношениями во многом определяется чувством любви. Прямую, хотя и слабую связь с удовлетворенностью показывает стиль «Сторге», который характеризует в большей мере не романтическую любовь как таковую, а стабильные дружеские или партнерские отношения.

Отсутствие связи стиля «Мания» с удовлетворенностью отношениями объясняется, по-видимому, противоречивыми как позитивными, так и негативными переживаниями (ревность, страх отвержения), характерными для этого стиля. Слабую обратную связь с удовлетворенностью показывает стиль «Прагма», проявляющийся в прагматичном отношении к партнеру, рациональности и расчетливости. Этот факт, вероятно, является следствием того, что рациональный учет достоинств и недостатков партнера не компенси- рует нехватку эмоциональной привязанности к нему и способствует разочарованию в отношениях.

Обратная связь стиля «Людус» с удовлетворенностью вполне ожидаема ввиду того, что безответственность и поверхностность в отношениях с партнером не предполагают глубоких чувств и стремления к поддержанию и развитию отношений. Этот стиль скорее свидетельствует об отсутствии любви в ее традиционном понимании, вместо которой имеет место несерьезная «игра в любовь» (Murthy et al., 1996).

Шкалы стилей любви являются тесно взаимозависимыми, так что их корреляционные связи с удовлетворенностью отношениями не позволяют сделать вывода о самостоятельном вкладе того или иного стиля в благополучие отношений. Для выявления среди стилей любви независимых существенных предикторов удовлетворенности отношениями был выполнен пошаговый множественный 
регрессионный анализ с исключением (таблица 4).

В результате анализа была получена регрессионная модель, объясняющая $32 \%$ дисперсии удовлетворенности отношениями. В соответствии с этой моделью сильный положительный вклад в удовлетворенность отношениями вносит стиль любви «Эрос», в то время как стиль «Людус» демонстрирует умеренную обратную связь с удовлетворенностью. Следовательно, эти два стиля являются наиболее существенными самостоятельными предикторами удовлетворенности.

Полученные результаты соответствуют данным, полученным в зарубежных исследованиях. В частности, в исследовании Г. Морроу было показано, что как для мужчин, так и для женщин характерна прямая связь удовлетворенности отношениями со стилями любви «Эрос», «Агапе» и «Мания» при наличии обратной связи со стилем «Людус» (Morrow et al., 1995). Интересно, что стили любви партнера в этом исследовании также показали аналогичные связи с удовлетворенностью, хотя сила связи была существенно меньше. В исследовании С. Хендрик с помощью регрессионного анализа результатов опроса 57 студенческих пар был получен подобный результат, свидетельствующий о том, что именно «Эрос» и «Людус» являются существенными предикторами удовлетворенности как для мужчин, так и для женщин (Hendrick, 1988). Таким образом, проведенный анализ стилей любви как предикторов удовлетворенности отношениями привел к ожидаемым результатам, что свидетельствует в пользу конструктной валидности русскоязычной версии шкалы оценки отношений.

Наряду со стилями любви к числу наиболее важных предикторов удовлетворенности близкими отношениями можно отнести также стили привязанности. Одним из наиболее известных теоретических подходов в исследовании стилей привязанности взрослых является концепция, предложенная К. Бартоломью и Л. Горовиц (Bartholomew, Horowitz, 1991). В основу этой концепции была положена идея Дж. Боулби о внутренней рабочей модели привязанности, складывающейся из двух составляющих. Первая из них характеризует обобщенную модель другого, вторая - обобщенную модель себя.

Таблица 4

Результаты множественного регрессионного анализа стилей любви как предикторов удовлетворенности отношениями $(\mathrm{N}=285)$

\begin{tabular}{|l|c|c|c|c|}
\hline & $\boldsymbol{\beta}$ & $\begin{array}{c}\text { Стдандартная ошибка } \\
\text { коэффициента } \boldsymbol{\beta}\end{array}$ & $\boldsymbol{t} \mathbf{( d f}=\mathbf{2 8 2})$ & $\boldsymbol{p}$ \\
\hline Св. член & & & 9.197 & $<.01$ \\
\hline Эрос & .512 & .049 & 1.382 & $<.01$ \\
\hline Людус & -.195 & .049 & -3.958 & $<.01$ \\
\hline
\end{tabular}

Примечание. $R=0.57 ; R^{2}=0.32 ; \mathrm{F}(2,282)=66.397 ; p<.001$. 
В поведенческих терминах их интерпретируют как тревогу (или беспокойство) в межличностных отношениях и избегание близких отношений.

В данном исследовании для диагностики стиля привязанности взрослых использовался опросник Опыт близких отношений (Fraley et al., 2000) в адаптации Т.В. Казанцевой (2008). Эта методика включает в себя две шкалы: тревоги и избегания в межличностных отношениях, которые в различных сочетаниях образуют четыре стиля привязанности. Авторами методики рекомендуется в ходе анализа для большей точности опираться на количественные значения тревоги и избегания без определения стиля привязанности (Fraley et al., 2000). Коэффициенты надежности $\alpha$ Кронбаха для этих шкал составили .81 и .70 соответственно.

Корреляционный анализ показателей стиля привязанности и удовлетворенности отношениями свидетельствует о наличии достоверной обратной связи шкалы избегания с оценкой отношений $(r=-.54 ; p<.001$; $\mathrm{N}=108)$. Следовательно, для лиц с избегающим стилем привязанности характерна относительно меньшая удовлетворенность близкими отношениями. Этот вывод соответствует результатам зарубежных исследований, которые также свидетельствуют о негативном влиянии избегающего стиля привязанности на удовлетворенность отношениями (Towler, Stuhlmacher, 2013; Juhl et al., 2012). Однако, в отличие от зарубежных данных, не удалось выявить связи удовлетворенности с тревогой в меж- личностных отношениях $(r=-.04$; $p$ - незначим).

\section{Удолетворенность отношениями и субъективное благополучие}

Удовлетворенность отношениями может рассматриваться как частное, специфическое для определенной сферы жизни проявление удовлетворенности жизнью или субъективного благополучия. Неудивительно, что удовлетворенность отношениями и браком показывает в исследованиях достоверную связь с показателями субъективного благополучия (Scorsolini-Comin, dos Santos, 2012). В данном исследовании для проверки гипотезы о связи удовлетворенности отношениями с общими показателями субъективного благополучия был проведен корреляционный анализ результатов по Шкале оценки отношений, Шкале субъективного благополучия А. Бадо и Г.А. Мендельсон и Шкале счастья С. Любомирски (см. таблицу 5). При интерпретации результатов следует учитывать тот факт, что Шкала субъективного благополучия является обратной, т.е. о высоком благополучии свидетельствуют низкие количественные значения.

Полученные коэффициенты корреляции полностью подтверждают гипотезу о наличии связи между удовлетворенностью отношениями и уровнем субъективного благополучия. Эти результаты свидетельствуют в пользу вывода о том, что Шкала оценки отношений действительно измеряет удовлетворенность отношениями как одну из составляющих удовлетворенности жизнью. 
Таблица 5

Коэффициенты корреляции Пирсона между удовлетворенностью отношениями и показателями субъективного благополучия $(\mathrm{N}=47)$

\begin{tabular}{|l|c|}
\hline \multicolumn{1}{|c|}{ Методика/шкала } & Шкала оценки отношений \\
\hline \multicolumn{2}{|c|}{ Субшкалы Шкалы субъективного благополучия } \\
\hline 1. Напряженность и чувствительность & $-.33^{*}$ \\
\hline $\begin{array}{l}\text { 2. Признаки, сопровождающие основную } \\
\text { психиатрическую симптоматику }\end{array}$ & $-.43^{* *}$ \\
\hline 3. Изменения настроения & -.17 \\
\hline 4. Значимость социального окружения & -.14 \\
\hline 5. Самооценка здоровья & -.07 \\
\hline $\begin{array}{l}\text { 6. Степень удовлетворенности повседневной } \\
\text { деятельностью }\end{array}$ & $-.40^{* *}$ \\
\hline Шкала субъективного благополучия & $-.45^{* *}$ \\
\hline Шкала счастья С. Любомирски & $.43^{* *}$ \\
\hline
\end{tabular}

${ }^{*} p<.05,{ }^{* *} p<.01$.

Влияние сочиальной желательности на результать методики

Высокая удовлетворенность может рассматриваться некоторыми лицами как социально значимая характеристика отношений. В этой связи можно предполагать, что результаты оценки отношений могут быть в некоторой степени искажены ввиду сознательной или неосознанной тенденции испытуемого представить себя в социально желательном свете. Для анализа влияния тенденции к социальной желательности на результаты диагностики удовлетворенности отношениями был использован опросник социальной желательности BIDR в адаптации E.Н. Осина. На выборке студентов $(\mathrm{N}=47)$ было проведено соответствующее исследование и вычисле- ны коэффициенты корреляции удовлетворенности отношениями со шкалами социальной желательности. В результате была выявлена достоверная связь оценки отношений со шкалой «Самообман - отрицание» $(r=.31 ; p<.05)$. Анализ корреляций отдельных заданий со шкалами социальной желательности показывает, что связь с самообманом характерна только для утверждений 5 и 7. Их содержание связано с оценкой соответствия отношений ожиданиям и количества проблем в отношениях. Это значит, что склонность к отрицанию проявляется в данном случае в недооценке расхождений между ожиданиями и реальностью и в преуменьшении проблем. Полученные результаты свидетельствует о том, что даже при условии анонимного предъявления методик результаты диагностики по Шкале оценки отношений могут 
быть подвержены эффектам социальной желательности, что необходимо учитывать при организации исследований.

\section{Социально-демографические} характеристики и

\section{удовлетворенность отношениями}

Анализ статистического распределения данных по Шкале оценки отношений свидетельствует о достоверном отличии от нормального (критерий Колмогорова-Смирнова $d=0.08, p<.01$; Лиллиефорса $p<.01)$. Распределение характеризуется явной левосторонней асимметрией $($ As $=-0.74)$, преобладанием относительно высоких значений по шкале, что является типичным для многих шкал, оценивающих показатели благополучия и удовлетворенности жизнью.

Представленные в таблице 6 результаты свидетельствуют о наличии статистически значимых различий между мужчинами и женщинами по шкале удовлетворенности отношениями, однако размер эффекта, равный 0.25, указывает на их относительно малую величину. Вывод о большей удовлетворенности мужчин соответствует результатам отечественных исследований удовлетворенности браком (Андреева, 2005;
Сопун, Лидерс, 2007), в то время как зарубежные данные о гендерных различиях в удовлетворенности отношениями противоречивы (Hendrick et al., 1998). Достоверных различий между средними значениями у лиц, состоящих в браке $(\mathrm{M}=3.79)$ и в добрачных отношениях ( $\mathrm{M}=3.77)$, не обнаружено.

Для проверки гипотезы о взаимодействии факторов пола и семейного положения был проведен двухфакторный дисперсионный анализ, результаты которого показали достоверные эффекты фактора пола, а также взаимодействия факторов пола и семейного положения ( $\mathrm{F}(1$, $598)=5.92 ; p<.05)$. Приведенные в таблице 7 средние значения по соответствующим подгруппам свидетельствуют о том, что удовлетворенность женщин практически одинакова в добрачных и супружеских отношениях, в то время как удовлетворенность мужчин, состоящих в браке, существенно выше, чем у мужчин, состоящих в добрачных отношениях.

В данном исследовании подтвердился факт нелинейной зависимости удовлетворенности отношениями от их стажа, что было ранее показано в исследованиях ряда зарубежных и отечественных авторов на супружеских парах (Walker, 1977; Алешина, 1985).

Таблица 6

Гендерные различия по удовлетворенности отношениями

\begin{tabular}{|c|c|c|c|c|c|c|c|c|}
\hline \multicolumn{2}{|c|}{$\begin{array}{l}\text { Мужчины } \\
(\mathrm{N}=176)\end{array}$} & \multicolumn{2}{|c|}{$\begin{array}{l}\text { Женщины } \\
(\mathrm{N}=426)\end{array}$} & \multicolumn{2}{|c|}{$\begin{array}{c}\text { Вся } \\
\text { выборка } \\
(\mathrm{N}=602)\end{array}$} & \multirow[t]{2}{*}{$\begin{array}{c}\text { U критерий } \\
\text { Манна-Уитни }\end{array}$} & \multirow[t]{2}{*}{$\begin{array}{c}t \\
(\mathrm{df}=600)\end{array}$} & \multirow{2}{*}{$\begin{array}{c}\text { Размер } \\
\text { эффекта } \\
\text { (d Коэна) }\end{array}$} \\
\hline M & $\mathrm{SD}$ & $\mathrm{M}$ & $\mathrm{SD}$ & M & $\mathrm{SD}$ & & & \\
\hline 3.92 & 0.61 & 3.72 & 0.84 & 3.78 & 0.79 & $33419(p<.05)$ & $2.76(p<.01)$ & 0.25 \\
\hline
\end{tabular}


Согласно Ю.Е. Алешиной, в течение первых двух десятилетий удовлетворенность браком постепенно понижается, достигая своего минимального значения в парах со стажем семейной жизни от 12 до 18 лет, а затем снова возрастает.

Результаты исследования (таблица 8) свидетельствуют о том, что в группе со стажем отношений от 4 до 15 лет происходит снижение удовлетворенности, однако затем ее уро- вень повышается ( $\mathrm{F}=2.85 ; p<.05)$. Вместе с тем сила влияния стажа очень мала: коэффициент эта-квадрат, отражающий долю объясняемой дисперсии, составляет всего лишь $1.4 \%$. Аналогичная тенденция обнаруживается и в том случае, если для анализа использовать только выборку лиц, состоящих в официальном браке, однако ввиду сокращения объема выборки этот эффект уже не имеет статистической значимости.

Таблица 7

Средние значения удовлетворенности отношениями в подгруппах разного пола и семейного положения

\begin{tabular}{|l|l|c|c|}
\hline \multicolumn{1}{|c|}{ Пол } & Семейное положение & Среднее значение (N) & Стд. отклонение \\
\hline Женский & Не замужем & $3.76(155)$ & 0.78 \\
\hline Женский & Замужем & $3.70(271)$ & 0.87 \\
\hline Мужской & Не женат & $3.78(89)$ & 0.61 \\
\hline Мужской & Женат & $4.06(87)$ & 0.59 \\
\hline
\end{tabular}

Таблица 8

Результаты анализа влияния возраста и стажа отношений на удовлетворенность

\begin{tabular}{|c|c|c|c|c|c|c|}
\hline $\begin{array}{c}\text { Фактор/ } \\
\text { группа }\end{array}$ & $\mathbf{N}$ & $\begin{array}{c}\text { Среднее } \\
\text { значение }\end{array}$ & $\begin{array}{c}\text { Стандартное } \\
\text { отклонение }\end{array}$ & $\begin{array}{c}\text { Критерий } \\
\text { Краскела- } \\
\text { Уоллиса Н } \\
(3, \mathbf{N}=602)\end{array}$ & $\begin{array}{c}\text { ANOVA } \\
\text { F(3, 598) }\end{array}$ & $\eta^{2}$ \\
\hline \multicolumn{4}{|c|}{ Возрастная группа } & 3.76 & 1.04 & .005 \\
\hline 17-25 лет & 355 & 3.79 & 0.73 & & & \\
\hline 26-34 лет & 143 & 3.70 & 0.88 & & & \\
\hline 35-43 лет & 70 & 3.84 & 0.83 & & & \\
\hline 44-60 лет & 34 & 3.93 & 0.84 & & & \\
\hline \multicolumn{4}{|c|}{ Стаж отношений } & $8.58^{*}$ & $2.85^{*}$ & .014 \\
\hline менее 1 года & 125 & 3.74 & 0.63 & & & \\
\hline 1-3 года & 202 & 3.89 & 0.73 & & & \\
\hline 4-15 лет & 214 & 3.68 & 0.90 & & & \\
\hline 16-40 лет & 61 & 3.88 & 0.80 & & & \\
\hline
\end{tabular}

$* p<.05$. 
С целью исследования возможного влияния пола и семейного положения на функционирование отдельных заданий шкалы был проведен DIF-анализ средствами программы Mplus 6 (Wang, Wang, 2012). В ходе анализа исходная модель шкалы (см. рисунок 1) была дополнена двумя переменными (пол и семейное положение) с их путями на общий фактор и каждое из заданий. Пол не показал статистически значимых путей на отдельные задания, выступая при этом в качестве достоверного предиктора удовлетворенности отношениями. Семейное положение продемонстрировало значимые пути на задания 3,5 и 6, при том что его влияние на фактор удовлетворенности отношениями не является статисти- чески значимым. Полученная в итоге модель приведена на рисунке 2.

Результаты DIF-анализа показывают, что, хотя методика разрабатывалась как единое средство диагностики удовлетворенности романтическими отношениями разного типа, на самом деле существуют некоторые тенденции в оценке по отдельным заданиям, характерные для лиц, состоящих в добрачных и в супружеских отношениях. В частности, испытуемые, состоящие в браке, склонны давать более высокие оценки по заданиям 3, 5 и 6 при равном уровне удовлетворенности отношениями. Следовательно, сравнение результатов лиц, состоящих в отношениях разного типа, требует известной осторожности, так как некоторые

\section{Влияние пола и семейного положения на результаты Шкалы оценки отношений}

Рисунок 2

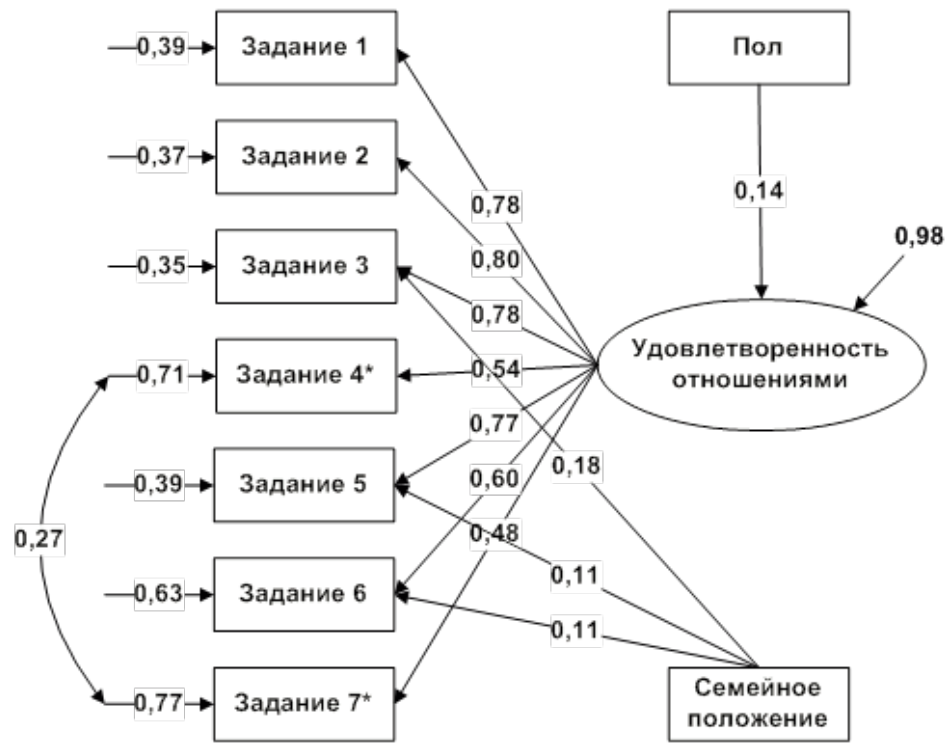

Примечание. Характеристики модели: $\chi^{2}=79.82 ; \mathrm{df}=24 ; p<.001$; RMSEA $=.062 ; 90 \%$-й доверительный интервал для RMSEA = .047-.078; CFI = .960; TLI = .941. Пол: $1-$ мужской, 0 - женский; семейное положение: 1 - женат/замужем, 0 - не женат/не замужем. 
утверждения, по-видимому, приобретают в различных отношениях разные оттенки смысла.

\section{Выводы}

Русскоязычная версия Шкалы оценки отношений представляет собой экономичную и гибкую методику диагностики удовлетворенности близкими (добрачными, супружескими и др.) отношениями. Данная методика имеет однофакторную структуру и характеризуется отличными показателями надежности по внутренней согласованности и ретестовой надежности. О хорошей конвергентной валидности свидетельствуют сильные связи показателей Шкалы оценки отношений с двумя другими методиками диагностики удовлетворенности браком. Полученные результаты свидетельствуют о полной взаимозаменяемости этих методик. Данные о кон- структной валидности Шкалы оценки отношений, полученные при сопоставлении ее результатов с показателями стилей любви, привязанности, субъективного благополучия и счастья, полностью соответствуют основанным на теории ожиданиям, а также результатам зарубежных исследований.

Таким образом, можно сделать вывод об отличных психометрических характеристиках русскоязычной версии Шкалы оценки отношений. Широкие возможности ее применения в исследовательской практике определяются актуальностью и востребованностью психологических и социологических исследований супружеских и добрачных отношений, гибкостью и методологической обоснованностью данной методики, а также наличием версий на разных языках, что открывает новые возможности для кросс-культурных исследований.

\section{Литература}

Алешина, Ю. Е. (1985). Удовлетворенность браком и межличностное восприятие в супружеских парах с различным стажем совместной жизни (Кандидатская диссертация, МГУ им. М.В. Ломоносова, Москва).

Алешина, Ю. Е., Гозман, Л. Я., Дубовская, Е. М. (1987). Социально-психологические методы исследования супружеских отношений: Спецпрактикум по социальной психологии. М.: Изд-во Московского ун-та.

Андреева, Т. В. (2005). Психология современной семьи. СПб.: Речь.

Екимчик, О. А. (2009). Когнитивный и эмоциональный компоненты любви у людей разного возраста (Кандидатская диссертация, Костромской государственный университет им. Н.А. Некрасова, Кострома).

Казанцева, Т. В. (2008). Адаптация модифицированной методики «Опыт близких отношений» К. Бреннан и Р. К. Фрейли. Известия Российского государственного педагогического университета им. А.И. Гериена, 34(74-2), 139-143.

Осин, Е. Н. (2011). Проблема социальной желательности в исследованиях личностного потенциала. В кн. Д. А. Леонтьев (Ред.), Личностный потенциал: структура и диагностика (с. 454-468). М.: Смысл. 
Осин, Е. Н., Леонтьев, Д. А. (2008). Апробация русскоязычных версий двух шкал экспресс-оценки субъективного благополучия. В кн. Материалы ІІІ Всероссийского социологического конгресса. М.: Институт социологии РАН/Российское общество социологов. Режим доступа: http://publications.hse.ru/en/chapters/78753840

Рюриков, Ю. Б. (1997). Цвета любви: от страсти до игры. М.: Рипол Классик.

Соколова, М. В. (1996). Шкала субъективного благополучия. Ярославль: НПЦ «Психодиагностика».

Сопун, С. М., Лидерс, А. Г. (2007). Психологическая совместимость супружеской пары и удовлетворенность браком. Сибирский психологический журнал, 25, 156-162.

Столин, В. В., Романова, Т. А., Бутенко, Г. П. (1984). Опросник удовлетворенности браком. Вестник Московского университета. Серия 14. Психология, 2, 54-60.

Фер, Р. М., Бакарак, В. Р. (2010). Психометрика: введение. Челябинск: Изд. центр ЮУрГУ.

Bartholomew, K., \& Horowitz, L. M. (1991). Attachment styles among young adults: a test of a 4-category model. Journal of Personality and Social Psychology, 61(2), 226-244. doi:10.1037/0022-3514.61.2.226

Cassepp-Borges, V., \& Pasquali, L. (2011). Relationship Assessment Scale's psychometric characteristics. Psico-USF, 16(3), 255-264. doi:10.1590/S1413-82712011000300002

Dinkel, A., \& Balck, F. (2005). An evaluation of the German relationship assessment scale. Sroiss Journal of Psychology/Schweizerische Zeitschrift für Psychologie/Revue Suisse de Psychologie, 64(4), 259-263. doi:10.1024/1421-0185.64.4.259

Fraley, R. C., Waller, N. G., \& Brennan, K. A. (2000). An item response theory analysis of self-report measures of adult attachment. Journal of Personality and Social Psychology, 78(2), 350-365. doi:10.1037/0022-3514.78.2.350

Guerrero, L. K., Anderson, P. A., \& Afifi, W. A. (2011). Close encounter: Close communication in relationships. Los Angeles: Sage.

Hatfield, E., Bensman, L., \& Rapson, R. L. (2012). A brief history of social scientists' attempts to measure passionate love. Journal of Social and Personal Relationships, 29(2), 143-164. doi:10.1177/0265407511431055

Hendrick, S. S. (1988). A generic measure of relationship satisfaction. Journal of Marriage and the Family, 50(1), 93-98. doi:10.2307/352430

Hendrick, S. S., Dicke, A., \& Hendrick, C. (1998). The relationship assessment scale. Journal of Social and Personal Relationships, 15(1), 137-142. doi: 10.1177/0265407598151009

Hendrick, S. S., \& Hendrick, C. (1997). Love and satisfaction. In R. J. Sternberg \& M. Hojjat (Eds.), Satisfaction in close relationships (pp. 56-78). New York/London: The Guilford Press.

Juhl, J., Sand, E. C., \& Routledge, C. (2012). The effects of nostalgia and avoidant attachment on relationship satisfaction and romantic motives. Journal of Social and Personal Relationships, 29(5), 661-670. doi:10.1177/0265407512443433

Koski, L. R., \& Shaver, P. R. (1997). Attachment and relationship satisfaction across the lifespan. In R. J. Sternberg \& M. Hojjat (Eds.), Satisfaction in close relationships (pp. 26-55). New York/London: The Guilford Press.

Morrow, G. D., Clark, E. M., \& Brock, K. F. (1995). Individual and partner love styles: Implications for the quality of romantic involvements. Journal of Social and Personal Relationships, 12(3), 363-387. doi:10.1177/0265407595123003

Murthy, K., Rotzien, A., \& Vacha-Haase, T. (1996). Validity studies second-order structure underlying the Hendrick-Hendrick Love Attitudes Scale. Educational and Psychological Measurement, 56(1), 108-121. doi:10.1177/0013164496056001007 
Rask, M., Malm, D., Kristofferzon, M. L., Roxberg, A., Svedberg, P., Arenhall, E., ... Benzein, E. (2009). Validity and reliability of a Swedish version of the Relationship Assessment Scale (RAS): A pilot study. Canadian Journal of Cardiovascular Nursing - Journal Canadien en Soins Infirmiers Cardiovasculaires, 20(1), 16-21.

Renshaw, K. D., McKnight, P., Caska, C. M., \& Blais, R. K. (2011). The utility of the relationship assessment scale in multiple types of relationships. Journal of Social and Personal Relationships, 28(4), 435-447. doi:10.1177/0265407510377850

Rusbult, C. E., \& Buunk, B. P. (1993). Commitment processes in close relationships: An interdependence analysis. Journal of Social and Personal Relationships, 10(2), 175-204. doi:10.1177/ 026540759301000202

Scorsolini-Comin, F., \& dos Santos, M. A. (2012). Correlations between subjective well-being, dyadic adjustment and marital satisfaction in Brazilian married people. The Spanish Journal of Psychology, 15(01), 166-176. doi:10.5209/rev_SJOP.2012.v15.n1.37304

Towler, A. J., \& Stuhlmacher, A. F. (2013). Attachment styles, relationship satisfaction, and well-being in working women. The Journal of Social Psychology, 153(3), 279-298. doi:10.1080/ 00224545.2012 .735282

Vaughn, M. J., \& Matyastik Baier, M. E. (1999). Reliability and validity of the relationship assessment scale. American Journal of Family Therapy, 27(2), 137-147. doi:10.1080/019261899262023

Walker, C. (1977). Some variations in marital satisfaction. In R. Chester \& J. Peel (Eds.), Equalities and inequalities in family life (pp. 127-139). London: Academic Press.

Wang, J., \& Wang, X. (2012). Structural equation modeling: applications using Mplus. Chichester, West Sussex, UK/Hoboken, NJ: John Wiley/Higher Education Press.

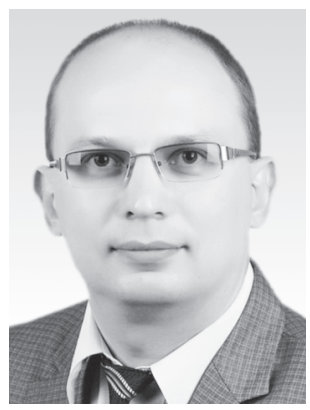

Сычев Олег Анатольевич - доцент, кафедра психологии, ФГБОУ ВПО «Алтайская государственная академия образования имени B.M. Шукшина», кандидат психологических наук. E-mail: osn1@mail.ru 


\section{Шкала оценки отношений}

Приложение

Инструкция. Приведенные ниже утверждения касаются вашей удовлетворенности отношениями с партнером (супругом или супругой, другом или подругой). Пожалуйста, для каждого утверждения на бланке укажите наиболее соответствующий вам вариант ответа.

1. Насколько хорошо партнер соответствует вашим потребностям?

1

Плохо
2

3
4

Умеренно
5

Очень хорошо

2. В целом насколько вы удовлетворены вашими взаимоотношениями с партнером?

$$
1
$$

2

3

4

5
Умеренно

3. Насколько близки ваши отношения к идеальным?
2

Очень мало
3

Умеренно
4 5

4. Как часто вы жалеете о том, что вступили в эти отношения?

1 2

3

4

5
Никогда
Иногда
Очень часто

5. В какой мере ваши взаимоотношения соответствуют тому, что вы от них ожидали?
1
2
3
4
5

Очень мало

Умеренно

Полностью

6. Насколько сильно вы любите своего партнера?

1

Слабо
2$$
\text { ( }
$$$$
\text { - } 3
$$

$$
\text { Умеренно }
$$

4

5

Очень сильно

7. Как много проблем в ваших отношениях?

1

2

3

4

5

Очень мало

Очень много

Обратные задания, ответы по которым необходимо инвертировать: 4. 7. Общая сумма оценок по всем заданиям делится на 7, полученный итоговый показатель по шкале от 1 до 5 характеризует удовлетворенность партнеров отношениями. 


\title{
Russian Version of Relationship Assessment Scale
}

\author{
Oleg A. Sychev \\ ${ }^{a}$ Shukshin Altai State Academy of Education, 53 Korolenko str., Altai region, Biysk, 659333, Russian \\ Federation
}

\begin{abstract}
The article presents the results of development of the Russian version of the Relationship Assessment Scale by S. Hendrick. As distinct from the satisfaction with marriage test, well-known in our country, this scale is characterized by good usability, parsimony and applicability for diagnostics of satisfaction with both marital and other intimate relationships between adults. Approbation of the proposed Russian version was conducted on three samples (general size $\mathrm{N}=$ 602), including students, employees of state-funded organizations and Internet users of various ages (who took part in online-testing), who were officially married or in premarital relationships. The results of confirmatory factor analysis confirmed the one-dimensional structure of the scale (with the presence of covariance, which reflected the specifics of respondents' reaction to the reversed items of the scale). The scale showed excellent reliability in internal consistency (Cronbach's alpha .84 - .89) and retest reliability (> .7). Convergent validity is confirmed by high correlations with two diagnostic scales of satisfaction with marriage $\left(\mathrm{r}_{1}=.82, \mathrm{r}_{2}=.88\right)$. Construct validity was confirmed by foreseeable correlations with scales of styles of love, attachment, subjective wellbeing and happiness. Moderate correlation was found between the measure of the scale and propensity for self-deception. Significant influence on relationships satisfaction was shown by factors of gender, marital status and their interaction, wherein the highest level of satisfaction was characteristic of men in marriage. Though relationships satisfaction in men is significantly higher than in women, the range of difference is not large (Cohen's $d=0.25$ ). The analysis of differential functioning of the items of the scale showed their independence from gender and dependence from marital status, which may reflect different interpretation of some items by people in relationships of various types. The acquired results allow to recommend the suggested version of the scale as an instrument for evaluation of satisfaction with marital or other intimate relationships of adults.
\end{abstract}

Keywords: relationship satisfaction, relationship assessment scale, love styles, attachment styles, subjective well-being.

\section{References}

Aleshina, Yu. E. (1985). Udovletvorennost' brakom i mezhlichnostnoe vospriyatie v supruzheskikh parakh s razlichnym stazhem sozmestnoi zhizni [Satisfaction with marriage and interpersonal perception in married couples with various length of joint life] (PhD dissertation, Lomonosov Moscow State University, Moscow).

Aleshina, Yu. E., Gozman, L. Ya., \& Dubovskaya, E. M. (1987). Sotsial'no-psikhologicheskie metody issledovaniya supruzheskikh otnoshenii: Spetspraktikum po sotsial'noi psikhologii [Social psychological methods to study marital relationships: Special practicum in social psychology]. Moscow: Moscow University Press. 
Andreeva, T. V. (2005). Psikhologiya sovremennoi sem’i [The psychology of modern family]. Saint Petersburg: Rech'.

Bartholomew, K., \& Horowitz, L. M. (1991). Attachment styles among young adults: a test of a 4-category model. Journal of Personality and Social Psychology, 61(2), 226-244. doi:10.1037/00223514.61.2.226

Cassepp-Borges, V., \& Pasquali, L. (2011). Relationship Assessment Scale's psychometric characteristics. Psico-USF, 16(3), 255-264. doi:10.1590/S1413-82712011000300002

Dinkel, A., \& Balck, F. (2005). An evaluation of the German relationship assessment scale. Swiss Journal of Psychology/Schweizerische Zeitschrift für Psychologie/Revue Suisse de Psychologie, 64(4), 259-263. doi:10.1024/1421-0185.64.4.259

Ekimchik, O. A. (2009). Kognitivnyi i emotsional'nyi i komponenty lyubvi u lyudei raznogo vozrasta [Cognitive and emotional components of love in people of different age] ( $\mathrm{PhD}$ dissertation, Nekrasov Kostroma State University, Kostroma, Russian Federation).

Fraley, R. C., Waller, N. G., \& Brennan, K. A. (2000). An item response theory analysis of self-report measures of adult attachment. Journal of Personality and Social Psychology, 78(2), 350-365. doi:10.1037/0022-3514.78.2.350

Furr, R. M., \& Bacharach, V. R. (2010). Psikhometrika: Vvedenie [Psychometrics: Introduction]. Chelyabinsk: South Ural State University Publishing House. (Transl. of: Furr, R. M., \& Bacharach, V. R. (2008). Psychometrics: An introduction. Thousand Oaks, CA: Sage Publications).

Guerrero, L. K., Anderson, P. A., \& Afifi, W. A. (2011). Close encounter: Close communication in relationships. Los Angeles: Sage.

Hatfield, E., Bensman, L., \& Rapson, R. L. (2012). A brief history of social scientists' attempts to measure passionate love. Journal of Social and Personal Relationships, 29(2), 143-164. doi:10.1177/0265407511431055

Hendrick, S. S. (1988). A generic measure of relationship satisfaction. Journal of Marriage and the Family, 50(1), 93-98. doi:10.2307/352430

Hendrick, S. S., \& Hendrick, C. (1997). Love and satisfaction. In R. J. Sternberg \& M. Hojjat (Eds.), Satisfaction in close relationships (pp. 56-78). New York/London: The Guilford Press.

Hendrick, S. S., Dicke, A., \& Hendrick, C. (1998). The relationship assessment scale. Journal of Social and Personal Relationships, 15(1), 137-142. doi: 10.1177/0265407598151009

Juhl, J., Sand, E. C., \& Routledge, C. (2012). The effects of nostalgia and avoidant attachment on relationship satisfaction and romantic motives. Journal of Social and Personal Relationships, 29(5), 661-670. doi:10.1177/0265407512443433

Kazantseva, T. V. (2008). Adaptation of the modified technique "Experiences in Close Relationships" by K. Brennan and R. Fraley. Izvestia: Herzen University Journal of Humanities and Sciences, 34(74-2), 139-143.

Koski, L. R., \& Shaver, P. R. (1997). Attachment and relationship satisfaction across the lifespan. In R. J. Sternberg \& M. Hojjat (Eds.), Satisfaction in close relationships (pp. 26-55). New York/London: The Guilford Press.

Morrow, G. D., Clark, E. M., \& Brock, K. F. (1995). Individual and partner love styles: Implications for the quality of romantic involvements. Journal of Social and Personal Relationships, 12(3), 363-387. doi:10.1177/0265407595123003

Murthy, K., Rotzien, A., \& Vacha-Haase, T. (1996). Validity studies second-order structure underlying the Hendrick-Hendrick Love Attitudes Scale. Educational and Psychological Measurement, 56(1), 108-121. doi:10.1177/0013164496056001007 
Osin, E. N. (2011). Problema sotsial'noi zhelatel'nosti v issledovaniyakh lichnostnogo potentsiala [The issue of social desirability in studies on personal potential]. In D. A. Leontiev (Ed.), Lichnostnyi potentsial: struktura i diagnostika [Personal potential: Structure and diagnostics] (pp. 454-468). Moscow: Smysl.

Osin, E. N., \& Leontiev, D. A. (2008). Aprobatsiya russkoyazychnykh versii dvukh shkal ekspressotsenki sub"ektivnogo blagopoluchiya [Approbation of the Russian versions of the two scales for express-evaluation of subjective well-being]. In Materialy III Vserossiiskogo Sotsiologicheskogo Kongressa [Proceedings of the Third All-Russian Sociological Congress]. Moscow: Institute of Sociology of the Russian Academy of Sciences / Russian Society of Sociologists. Retrieved from http://publications.hse.ru/en/chapters/78753840

Rask, M., Malm, D., Kristofferzon, M. L., Roxberg, A., Svedberg, P., Arenhall, E., ... Benzein, E. (2009). Validity and reliability of a Swedish version of the Relationship Assessment Scale (RAS): A pilot study. Canadian Journal of Cardiovascular Nursing - Journal Canadien en Soins Infirmiers Cardiovasculaires, 20(1), 16-21.

Renshaw, K. D., McKnight, P., Caska, C. M., \& Blais, R. K. (2011). The utility of the relationship assessment scale in multiple types of relationships. Journal of Social and Personal Relationships, 28(4), 435-447. doi:10.1177/0265407510377850

Rusbult, C. E., \& Buunk, B. P. (1993). Commitment processes in close relationships: An interdependence analysis. Journal of Social and Personal Relationships, 10(2), 175-204. doi:10.1177/ 026540759301000202

Ryurikov, Yu. B. (1997). Tsveta lyubvi: ot strasti do igry [Colors of love: From passion to game]. Moscow: Ripol Klassik.

Sacher, J., \& Fine, M. (1996). Predicting relationship status and satisfaction after six months among dating couples. Journal of Marriage and the Family, 58(1), 21-32.

Scorsolini-Comin, F., \& dos Santos, M. A. (2012). Correlations between subjective well-being, dyadic adjustment and marital satisfaction in Brazilian married people. The Spanish Journal of Psychology, 15(01), 166-176. doi:10.5209/rev_SJOP.2012.v15.n1.37304

Sokolova, M. B. (1996). Shkala sub"ektionogo blagopoluchiya [The subjective wellbeing scale]. Yaroslavl: NPTs "Psikhodiagnostika".

Sopun, S. M., \& Liders, A. G. (2007). Psycological compability of a married cople and satisfaction of marriage. Siberian Journal of Psychology, 25, 156-162.

Stolin, V. V., Romanova, T. A., \& Butenko, G. P. (1984). Oprosnik udovletvorennosti brakom [The satisfaction with marriage questionnaire]. Vestnik Moskorskogo Universiteta. Seriya 14. Psikhologiya, 2, 54-60.

Towler, A. J., \& Stuhlmacher, A. F. (2013). Attachment styles, relationship satisfaction, and well-being in working women. The Journal of Social Psychology, 153(3), 279-298. doi:10.1080/ 00224545.2012 .735282

Vaughn, M. J., \& Matyastik Baier, M. E. (1999). Reliability and validity of the relationship assessment scale. American Journal of Family Therapy, 27(2), 137-147. doi:10.1080/019261899262023

Walker, C. (1977). Some variations in marital satisfaction. In R. Chester \& J. Peel (Eds.), Equalities and inequalities in family life (pp. 127-139). London: Academic Press.

Wang, J., \& Wang, X. (2012). Structural equation modeling: applications using Mplus. Chichester, West Sussex, UK/Hoboken, NJ: John Wiley/Higher Education Press.

Oleg A. Sychev - associate professor, Shukshin Altai State Academy of Education, Ph.D. E-mail: osn1@mail.ru 